\title{
LUCID upgrade for ATLAS luminosity measurement in Run II
}

\author{
Giulia Ucchielli*† \\ University of Bologna and INFN \\ E-mail: giulia.ucchielliecern.ch
}

\begin{abstract}
The main ATLAS luminosity monitor, LUCID, and its read-out electronics have been completely rebuilt for the LHC Run II in order to cope with a higher center of mass energy $(\sqrt{s}=13 \mathrm{TeV})$ and the $25 \mathrm{~ns}$ bunch-spacing. The LUCID detector is measuring Cherenkov light produced in photomultiplier quartz windows and in quartz optical fibers. It has a novel calibration system that uses radioactive ${ }^{207} \mathrm{Bi}$ sources that produce internal-conversion electrons with energy above the Cherenkov threshold in quartz. The new electronics can count signals with amplitude above a predefined threshold (hits) as well as the integrated pulseheight of the signals, which makes it possible to measure luminosity with complementary methods. The new detector, calibration system and electronics will be described, together with the results of the 2015 luminosity measurement.
\end{abstract}

38th International Conference on High Energy Physics

3-10 August 2016

Chicago, USA

\footnotetext{
* Speaker.

${ }^{\dagger}$ for the ATLAS Collaboration.
} 


\section{Introduction}

Luminosity $(\mathscr{L})$ is a key quantity for any physics measurement since it directly enters the cross-section calculation together with the observed number of events. The uncertainty on a luminosity measurement represents the largest systematics in many physics analyses. For this reason, ATLAS provides several measurements of $\mathscr{L}$ with LUCID as main detector and the calorimeters and the inner detector providing additional measurements for the systematic evaluation.

\section{Motivation for the upgrade}

After the Run I shutdown, LUCID [1] had to be redesigned in order to cope with the data taking conditions foreseen for Run II. The tackling of the following problems led to specific new design choices:

- The expected larger pile-up would have made the migration problem worse. Migration consists in spurious hits generated by the sum of signals which would be individually under threshold. In addition, some event counting algorithms were already close to saturation, or already saturated, in Run I. Saturation occurs when hits are recorded for all bunch crossings.

- The beam pipe material was changed from stainless steel to aluminium. Monte Carlo simulations showed that this would increase the number of particles hitting LUCID by a factor of 4.

- The bunch spacing reduction from $50 \mathrm{~ns}$ to $25 \mathrm{~ns}$.

The first two problems were solved in Run II by reducing the acceptance of the detector by using smaller photomultiplier diameters (from $15 \mathrm{~mm}$ to $10 \mathrm{~mm}$ ) [2]. The third problem required a redesign of the electronics. A new set of VME boards (LUCROD) digitize the signals close to the detector to avoid distortions and optimize discriminator performances.

\section{The LUCID II detector and electronics}

LUCID is composed of two modules (one of which is shown in Fig. 1) placed around the beam-pipe on both forward ends of ATLAS, $17 \mathrm{~m}$ from the interaction point (IP).

Each module is made of 20 PMTs by Hamamatsu (R760) arranged in 5 groups of independent sensors with different features: 4 PMTs fit with ${ }^{207} \mathrm{Bi}$ radioactive source for calibration purposes, 4 PMTs calibrated with LED signals, 4 reduced-window (partially opacified by a thin aluminium layer, in order to further reduce the acceptance) PMTs and 4 PMTs fed by quartz fiber and located in a lower radiation area a few meters away. In addition, a fifth spare detector is available.

The custom-made VME boards, installed $15 \mathrm{~m}$ away from the sensors, digitize signals (defining a hit if above a given threshold) and integrate the pulses every $25 \mathrm{~ns}$ (charge measurement). Both hit-counting and the charge measurement are thus provided at each LHC bunch crossing. 
Furthermore, two VME boards (LUMAT) receive information from the LUCRODs via optical links and correlate hits coming from the two sides of the detector, to provide coincidence algorithms. The main advantage of charge measurement is its insensitivity to migration, due to the absence of a threshold requirement. In addition, the total charge is directly proportional to luminosity and does not have to rely on Poisson statistics ${ }^{1}$. The new electronics allow to implement about 100 different (although not all independent) algorithms.

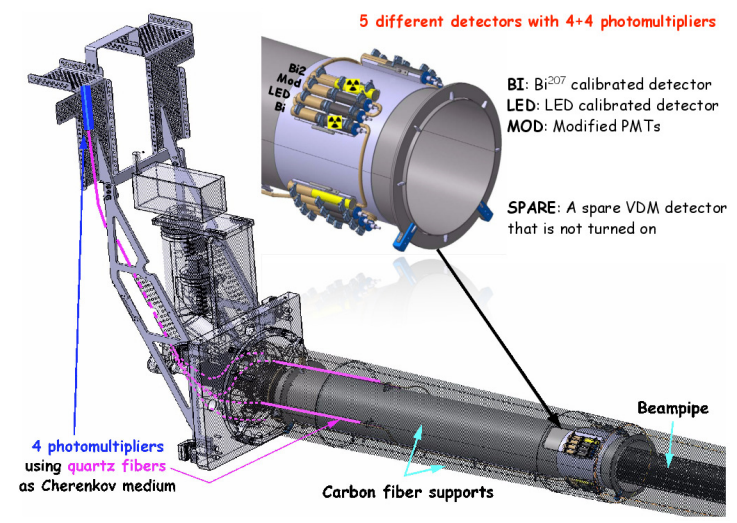

Figure 1: View of one of the two LUCID detector modules in 2016 [2].

\section{The calibration system}

Stability in the luminosity measurement at the \% level requires corrections for gain loss or ageing, both affecting the PMTs during the data taking.

The new LUCID calibration system is mainly based on ${ }^{207} \mathrm{Bi}$ sources. The PMTs which are not equipped with a radioactive source can be calibrated with LED signals. ${ }^{207} \mathrm{Bi}$ emission allows accurate calibration of the PMTs since the energy of the emitted electrons (around $1 \mathrm{MeV}$ ) mimics the signal of high energy charged particles crossing the same quartz window. The calibration procedure consists of monitoring the mean value of the charge distribution collected by PMTs from ${ }^{207} \mathrm{Bi}$ electrons. Figure 2 shows the percentage variation of the mean charge value,

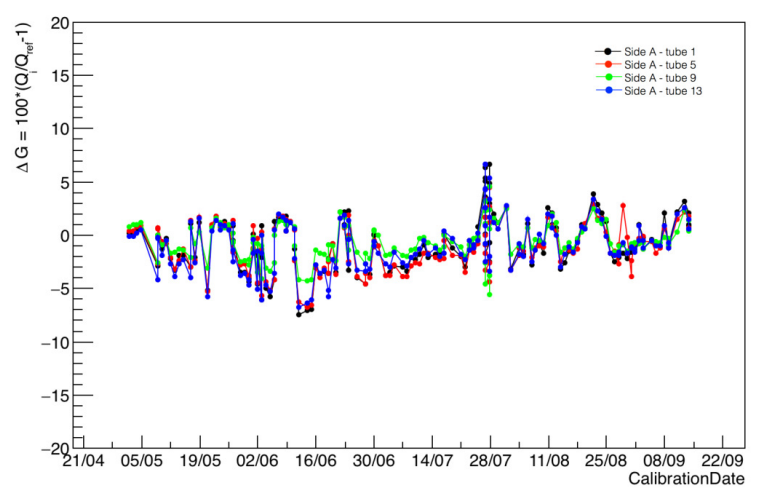

Figure 2: $\mathrm{Bi}^{207}$ calibration trending plot in 2016 . as a function of time, with respect to a reference run at the beginning of the data taking. Gain changes are aimed to be kept within 5\%, which reflects in a luminosity variation of about $1 \%$ for hit counting algorithms. Dedicated calibration sessions are performed at the end of each LHC fill and possible gain losses are automatically compensated through the increase of the high voltage to the PMTs. The success of the ${ }^{207} \mathrm{Bi}$ calibrations led to the installation of 4 new ${ }^{207} \mathrm{Bi}$ PMTs per side during the 2016 winter shutdown.

\section{Luminosity measurement in $\mathbf{2 0 1 5}$}

LUCID provides the official measurement of the ATLAS luminosity for physics analysis and the instantaneous luminosity to LHC, for beam stability monitoring, through event/hit counting

\footnotetext{
${ }^{1}$ The methods for luminosity measurement will be discussed in section 5 .
} 
algorithms. An event is defined as a particular hit configuration (i.e. hitOR or hitAND).

Hits and events can be related to the average number of interactions per bunch crossing $(\mu)$ via Poisson statistics. The luminosity, at each LB, can be measured by adding the luminosity for each individual bunch crossing:

$$
\mathscr{L}_{L B}=\frac{f_{L H C}}{\sigma^{\text {inel }}} \sum_{j=1}^{n_{b}} \mu_{j}=\frac{f_{L H C}}{\sigma^{v i s}} \sum_{j=1}^{n_{b}} \mu_{j}^{v i s}
$$

where $\sigma^{\text {vis }}$ is the absolute calibration constant obtained from dedicated LHC fills (called van der Meer scans), $f_{L H C}$ is the LHC revolution frequency, $n_{b}$ is the number of colliding bunches and $\mu^{v i s}$ is the measured mean number of interactions per bunch crossing. The LUCID measurement is monitored by the inner detector and the electromagnetic and hadronic tile calorimeters. The latter have been cross-calibrated to LUCID in a group of physics runs close to the vdM scan. The fractional difference between LUCID and the other detectors (Fig. 3) reveals a consistency between luminosity measurements as a function of time and shows a run to run stability at the level of $1 \%$ in 2015. This is one of the components of the overall systematic uncertainty in the luminosity measurement.

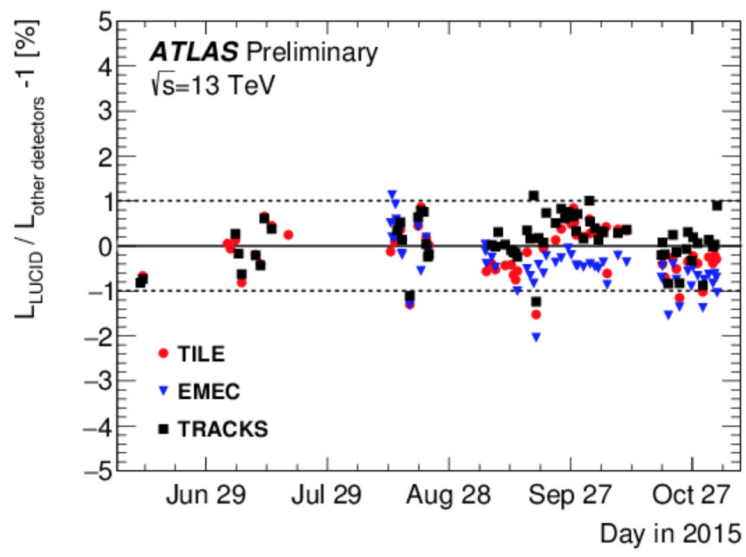

Figure 3: Run to run stability in 2015. Fractional difference with respect to LUCID is shown for the luminosity provided by the Inner Detector (black dots), the Tile (red circles) and the Electromagnetic calorimeters (blue triangles)[3].

The other main systematic uncertainties arise from the vdM calibration $(\sim 1.6 \%)$ and the calibration transfer $(\sim 0.9 \%)$ from low- $\mu$ (vdM) to high- $\mu$ (physics) runs. A final uncertainty of $2.1 \%$ has been achieved both for the $50 \mathrm{~ns}$ and $25 \mathrm{~ns}$ bunch spacing periods in 2015.

\section{References}

[1] ATLAS Collaboration, Improved luminosity determination in pp collisions at $\sqrt{s}=7 \mathrm{TeV}$ using the ATLAS detector at the LHC, Eur. Phys. J. C 73 (2013) 8, 2518 [arXiv:1302.4393 [hep-ex]].

[2] Choice and characterization of photomultipliers for the new ATLAS LUCID detector, G.L. Alberghi et al., Journal of Instrumentation, Volume 11; Number 5; p05014 (2016).

[3] https://twiki.cern.ch/twiki/bin/view/AtlasPublic/ LuminosityPublicResultsRun2\#Luminosity_summary_plots_for_AN1 